\title{
Magnetospherically reflected chorus waves revealed by ray tracing with CLUSTER data
}

\author{
M. Parrot ${ }^{1}$, O. Santolík ${ }^{2}$, N. Cornilleau-Wehrlin ${ }^{3}$, M. Maksimovic ${ }^{4}$, and C. Harvey ${ }^{5}$ \\ ${ }^{1}$ LPCE/CNRS, 3A Avenue de la Recherche, ORLEANS, 45071 France \\ ${ }^{2}$ Charles University, V. Holesovickach, Praha, 18000 Czech Republic \\ ${ }^{3}$ CETP/UVSQ, 10/12 Avenue de L'Europe, Velizy, 78140 France \\ ${ }^{4}$ LESIA, place Jules Janssen, Meudon, 92195 France \\ ${ }^{5}$ CESR, 9 Av. Colonel Roche, Toulouse, 31029 France
}

Received: 29 October 2002 - Revised: 20 February 2003 - Accepted: 4 March 2003

\begin{abstract}
This paper is related to the propagation characteristics of a chorus emission recorded simultaneously by the 4 satellites of the CLUSTER mission on 29 October 2001 between 01:00 and 05:00 UT. During this day, the spacecraft (SC) 1, 2, and 4 are relatively close to each other but SC3 has been delayed by half an hour. We use the data recorded aboard CLUSTER by the STAFF spectrum analyser. This instrument provides the cross spectral matrix of three magnetic and two electric field components. Dedicated software processes this spectral matrix in order to determine the wave normal directions relative to the Earth's magnetic field. This calculation is done for the 4 satellites at different times and different frequencies and allows us to check the directions of these waves. Measurements around the magnetic equator show that the parallel component of the Poynting vector changes its sign when the satellites cross the equator region. It indicates that the chorus waves propagate away from this region which is considered as the source area of these emissions. This is valid for the most intense waves observed on the magnetic and electric power spectrograms. But it is also observed on $\mathrm{SC} 1, \mathrm{SC} 2$, and $\mathrm{SC} 4$ that lower intensity waves propagate toward the equator simultaneously with the SC3 intense chorus waves propagating away from the equator. Both waves are at the same frequency. Using the wave normal directions of these waves, a ray tracing study shows that the waves observed by SC1, SC2, and SC4 cross the equatorial plane at the same location as the waves observed by SC3. $\mathrm{SC} 3$ which is 30 minutes late observes the waves that originate first from the equator; meanwhile, SC1, SC2, and SC4 observe the same waves that have suffered a Lower Hybrid Resonance (LHR) reflection at low altitudes (based on the ray tracing analysis) and now return to the equator at a different location with a lower intensity. Similar phenomenon is observed when all SC are on the other side of the equator. The intensity ratio between magnetic waves coming directly from the equator and waves returning to the equator is be-
\end{abstract}

Correspondence to: M. Parrot

(mparrot@cnrs-orleans.fr) tween 0.005 and 0.01 , which is in agreement with previously published theoretical calculation of the growth rates with the particle distribution seen by GEOS.

Key words. Magnetospheric physics (plasma waves and instabilities) - Ionosphere (wave propagation) - Radio science (magnetospheric physics)

\section{Introduction}

Chorus waves are one of the most intense emissions observed in the outer magnetosphere. They are detected near the equatorial plane and they are characterised by a sequence of discrete elements (rising and falling tones) with a time separation between 0.1 and $1 \mathrm{~s}$. They have been extensively studied in the past (see the review by Sazhin and Hayakawa (1992) and the references herein). They are generated by the injection of substorm electrons (Tsurutani and Smith, 1974; Tsurutani et al., 1979; Meredith et al., 2001) through the loss cone instability (Kennel and Petschek, 1966). It has been indirectly shown (Dunkel and Helliwell, 1969; Burtis and Helliwell, 1969; Burton and Holzer, 1974; Tsurutani and Smith, 1977) that the chorus emissions are generated near the magnetic equator. More recently, this was confirmed by Nagano et al. (1996) using GEOTAIL data and by LeDocq et al. (1998) with POLAR data. Parrot et al. (2003) have shown that in fact, during moderate magnetic activity, the source of the emissions is located at the magnetic equator when this equator is determined by the minimum of the in situ measured magnetic field (the SC velocity being parallel to this magnetic field). Around the magnetic equator, the frequency spectrum of chorus often shows that chorus have two distinct bands above and below one-half the electron gyrofrequency (Tsurutani and Smith, 1974). Up to now all observations have failed to find reflected chorus components returning to the equator. LeDocq et al. (1998) indicates that there is little evidence of reflected waves returning along the same field lines. Burton and Holzer (1974) and Goldstein and 
Tsurutani (1984) claim that the chorus emissions must encounter strong Landau damping as they propagate to high latitudes. The purpose of this paper is to analyse a chorus event where reflected components are observed using the wave experiment STAFF (Spatio-Temporal Analysis of Field Fluctuations) of the 4 CLUSTER satellites. STAFF will be shortly described in Sect. 2. Section 3 will present the dedicated software PRASSADCO (PRopagation Analysis of STAFFSA Data with COherency tests) used to determine propagation characteristics of the observed chorus waves, and the ray tracing program used to emulate the back and forth trajectories of the waves in the magnetosphere. The chorus event simultaneously recorded by the four CLUSTER satellites is described in Sect. 4 with the results of the ray tracing analysis. Discussions and conclusion are given in Sect. 5.

\section{The wave experiment}

The STAFF experiment (Cornilleau-Wehrlin et al., 1997, 2000, 2003) is a part of the Wave Experiment Consortium (WEC) (Pedersen et al., 1997) on board the four CLUSTER satellites. STAFF consists of three orthogonal magnetic search coils linked to a processing unit. Two sensors lie in the spin plane and the third one is parallel to the SC spin axis. The processing unit has two parts: STAFF-SC which records waveforms of the three components of the magnetic field up to $10 \mathrm{~Hz}(180 \mathrm{~Hz}$ in the burst mode), and STAFF-SA which computes the spectral matrix of five components of the electromagnetic field up to $4 \mathrm{kHz}$. The two electric components are from the Electric Field and Wave experiment EFW (Gustafsson et al., 2001). The SA (Spectrum Analyser) part will be used in the following. It produces $5 \times 5$ spectral matrix with 27 frequencies distributed logarithmically between $8 \mathrm{~Hz}$ and $4 \mathrm{kHz}$. This frequency range is divided into three frequency sub-bands: A $(8-64 \mathrm{~Hz}), \mathrm{B}(64-512 \mathrm{~Hz})$, and C $(512-4000 \mathrm{~Hz})$ with different time intervals of Fourier analysis (for example, for one second of data, 64 spectra are averaged in the band $\mathrm{C}, 8$ in the band $\mathrm{B}$, and only one spectrum is considered in band A). After averaging, the time resolution of the spectral matrix varies between $125 \mathrm{~ms}$ and $4 \mathrm{~s}$.

\section{The data processing software}

PRASSADCO is a computer program designed to analyse multicomponent measurements of electromagnetic waves. It implements a number of methods used to estimate polarisation and propagation parameters, such as the degree of polarisation, sense of elliptic polarisation and axes of polarisation ellipse, the wave vector direction, the Poynting vector, and the refractive index (Santolík and Parrot, 1998, 1999; Santolík, 2001). The main purpose of PRASSADCO is to facilitate scientific analysis of the spectral matrix obtained by the STAFF-SA instruments on board the four satellites. The inputs of this software are the CLUSTER data CD-ROMs provided by ESA, the CSDS (CLUSTER Science Data System) Prime Parameters (PP) of FGM (Flux Gate Magnetometer) for the local magnetic field (Balogh et al., 1997), and the CSDS Summary Parameters of auxiliary data (Daly, 2002). The results can be represented in different visual and numerical formats. The calculation of the wave normal directions relative to the Earth's magnetic field is done for the 4 satellites at different times and different frequencies according to the event we are looking at.

All these wave normal directions (two angles) obtained at a given frequency during the considered time interval are the inputs of a ray tracing program used to trace back all rays to the source. We use the three-dimensional procedure of Cerisier (1970) which was more recently used by Cairo and Lefeuvre (1986) and Muto and Hayakawa (1987). The ray tracing software uses a dipolar approximation of the magnetic field and a diffusive equilibrium model for ion and electron densities. The model is roughly calibrated at the satellite altitude by plasma parameters obtained by the WHISPER experiment (Décréau et al., 2001). Ray plots are represented in 3-D using a GSM (Geocentric Solar Magnetospheric) system.

\section{Detailed analysis of the event recorded by CLUSTER}

Figure 1 represents data recorded by the 4 spacecraft on 29 October 2001 between 01:00 and 06:00 UT. This event occurs during a period of moderate magnetic activity (DST is equal to $-65 \mathrm{nT}$ at 03:00 UT) just after a disturbed period. The first four panels show the magnetic power-spectral densities, for SC1, SC2, SC3, and SC4, respectively. The second four panels show the electric power-spectral densities, for SC1, SC2, SC3, and SC4, respectively. The plotted spectrograms are obtained from the arithmetic average of the power-spectral densities of the three magnetic components in the first four panels, and of the two electric components in the second four panels (the power-spectral densities themselves being already averaged (see Sect. 2)). A banded electromagnetic emission of chorus type is seen between $100 \mathrm{~Hz}$ and $4 \mathrm{kHz}$, with the maximum frequency being obtained when the satellite is near the magnetic equator. It has been shown by Dunkel and Helliwell (1969) and Burtis and Helliwell (1969) that the chorus emission frequency is related to the equatorial cyclotron frequency of the magnetic field line passing through the observing point. This explains why, in Fig. 1, the frequency of the chorus bands decreases on each side of the equator. In this event, chorus are observed below one-half the electron gyrofrequency. Besides the chorus emissions, on the one hand, turbulent electrostatic noise at low frequencies are observed in the auroral zones and close to the equator, and on the other hand, auroral hiss between $700 \mathrm{~Hz}$ and $4 \mathrm{kHz}$ are observed in the auroral zones. But these three emissions are not related to our study and they will not be considered here. The thin vertical white lines in the electric spectrograms are due to changes in the WEC experiment mode which temporarily inhibit the observation of natural waves during WHISPER sounding (Décréau et al., 1997). 


\section{CLUSTER STAFF-SA 2001-10-29 01:00:00.051 - 2001-10-29 05:59:59.971}

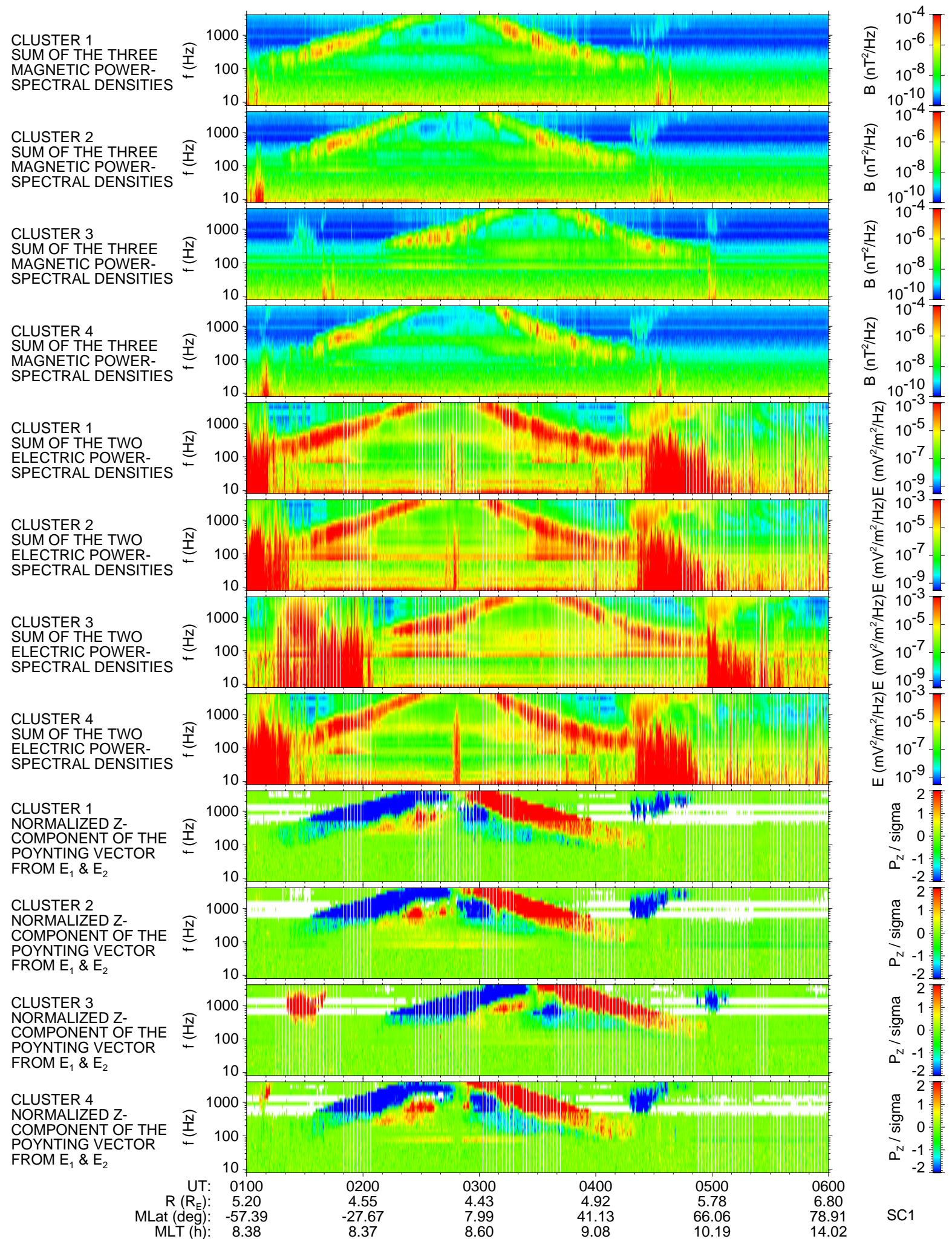

Fig. 1. Data recorded by the STAFF-SA experiment on 29 October 2001 between 01:00 and 06:00 UT. From the top to the bottom, the panels represent the magnetic power-spectral density, the electric power-spectral density, and the direction of the z-component of the Poynting vector, for each SC, respectively. The intensities of the spectrograms and the reliability of the sense of the Poynting vector (see Parrot et al., 2003) are given by the colour-coded scales on the right. The geophysical parameters at the bottom are related to SC1. 
CLUSTER STAFF-SA 2001-10-29 02:20:00.352 - 2001-10-29 03:49:59.658

CLUSTER 1

POLAR ANGLE

THETA

(Means, 1972)

CLUSTER 2

POLAR ANGLE

THETA

(Means, 1972)

CLUSTER 3

POLAR ANGLE

THETA

(Means, 1972)

CLUSTER 4

POLAR ANGLE

THETA

(Means, 1972)

\section{CLUSTER 1}

AZIMUTHAL

AZIMUTHAL

(Means, 1972)

CLUSTER 2

AZIMUTHAL

ANGLE PHI

(Means, 1972)

CLUSTER 3

AZIMUTHAL
ANGLE PHI

(Means, 1972)

CLUSTER 4

AZIMUTHAL

ANGLE PHI

(Means, 1972)

CLUSTER 1

NORMALIZED ZCOMPONENT OF THE POYNTING VECTOR $F R O M E_{1} \& E_{2}$

CLUSTER 2 NORMALIZED Z COMPONENT OF THE POYNTING VECTOR FROM $E_{1} \& E_{2}$

\section{CLUSTER 3} NORMALIZED Z COMPONENT OF THE POYNTING VECTOR FROM $E_{1} \& E_{2}$

CLUSTER 4 NORMALIZED ZCOMPONENT OF THE POYNTING VECTOR $F R O M E_{1} \& E_{2}$
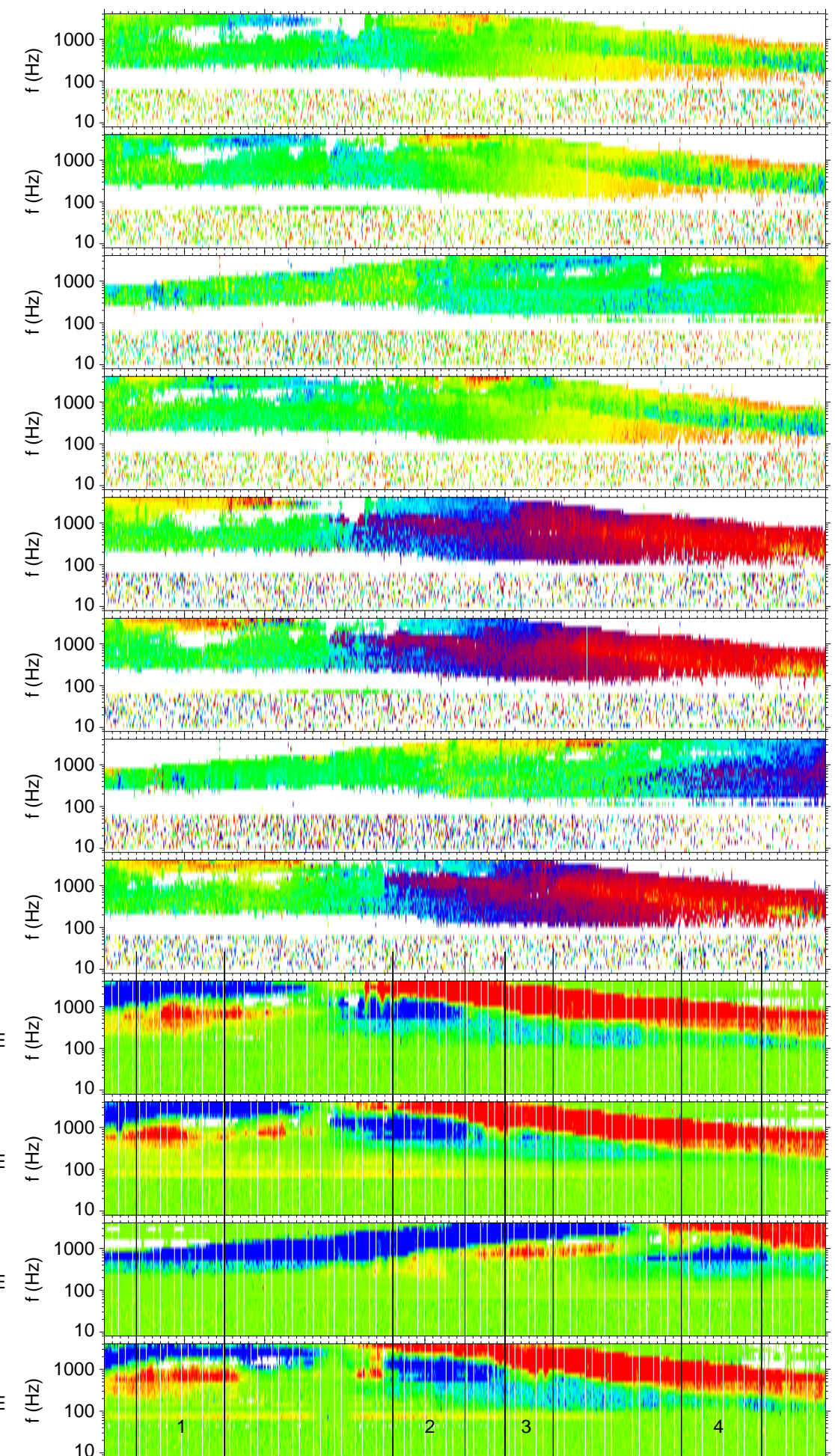
UT:
$R\left(\mathrm{R}_{\mathrm{E}}\right)$ :
MLat $(\mathrm{deg})$ :

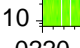
MLT (h):

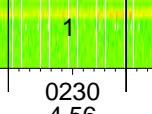

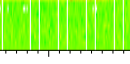
$\begin{array}{ccc}4.56 & 4.46 & 4.39 \\ -35.33 & -29.62 & -23.68\end{array}$

8.28

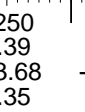

68

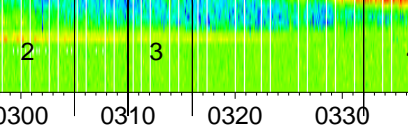

\begin{tabular}{l|l|l}
0300 & 0310 & 0320
\end{tabular}

$\begin{array}{ccc}4.33 & 4.28 & 4.26 \\ -1.55 & -11.27 & -4.88 \\ 8.40 & 8.45 & 8.51\end{array}$

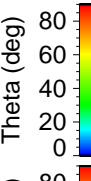

क्षे 80

त 40

$\stackrel{\Phi}{\lessgtr} 20$

कิ 80

응 60

ㄴ 40

$\stackrel{ \pm}{\rightleftarrows} 20$

क्ष 80

\% 60

要 40

$\stackrel{ \pm}{\lessgtr} 20$

कิ 90

$\begin{array}{lr}\text { के } & 90 \\ \frac{\Phi}{0} & 0\end{array}$

름 -90

$-180$

क्ष 90 毒

$\overline{\frac{\bar{c}}{\alpha}}-90$

$-180$

180

क्षे 90

둠

क -90

कิ $\quad 90$ 章

$\begin{array}{lr}\frac{d}{0} & 90 \\ \frac{\bar{c}}{\alpha} & -90\end{array}$

-90
-180
-2

$\begin{array}{ll}\frac{\pi}{E} & 2 \\ \frac{0}{\omega} & 0\end{array}$

$\Omega^{N}-1$

-2
2
1

हึ 1

क 0

$\alpha^{N}-1$

$-2$

Е్ర

哥 0

$\mathrm{Q}^{N}-1$

$\begin{array}{r}-2 \\ 2 \\ \hline\end{array}$

हू 1

क 0

$\Lambda^{N}-1$

-1
-2

Fig. 2. Zoom of the event of Fig. 1 between 02:20 and 03:50 UT. From the top to the bottom, the panels represent the polar angle $\theta$ and the azimuthal angle $\phi$ of the wave normal direction, and the direction of the $z$-component of the Poynting vector, for each SC, respectively. The values for the angles and the reliability of the sense of the Poynting vector are given by the colour-coded scales on the right. The geophysical parameters at the bottom are related to SC3. The vertical black lines delimit the four time intervals of detailed analysis. 

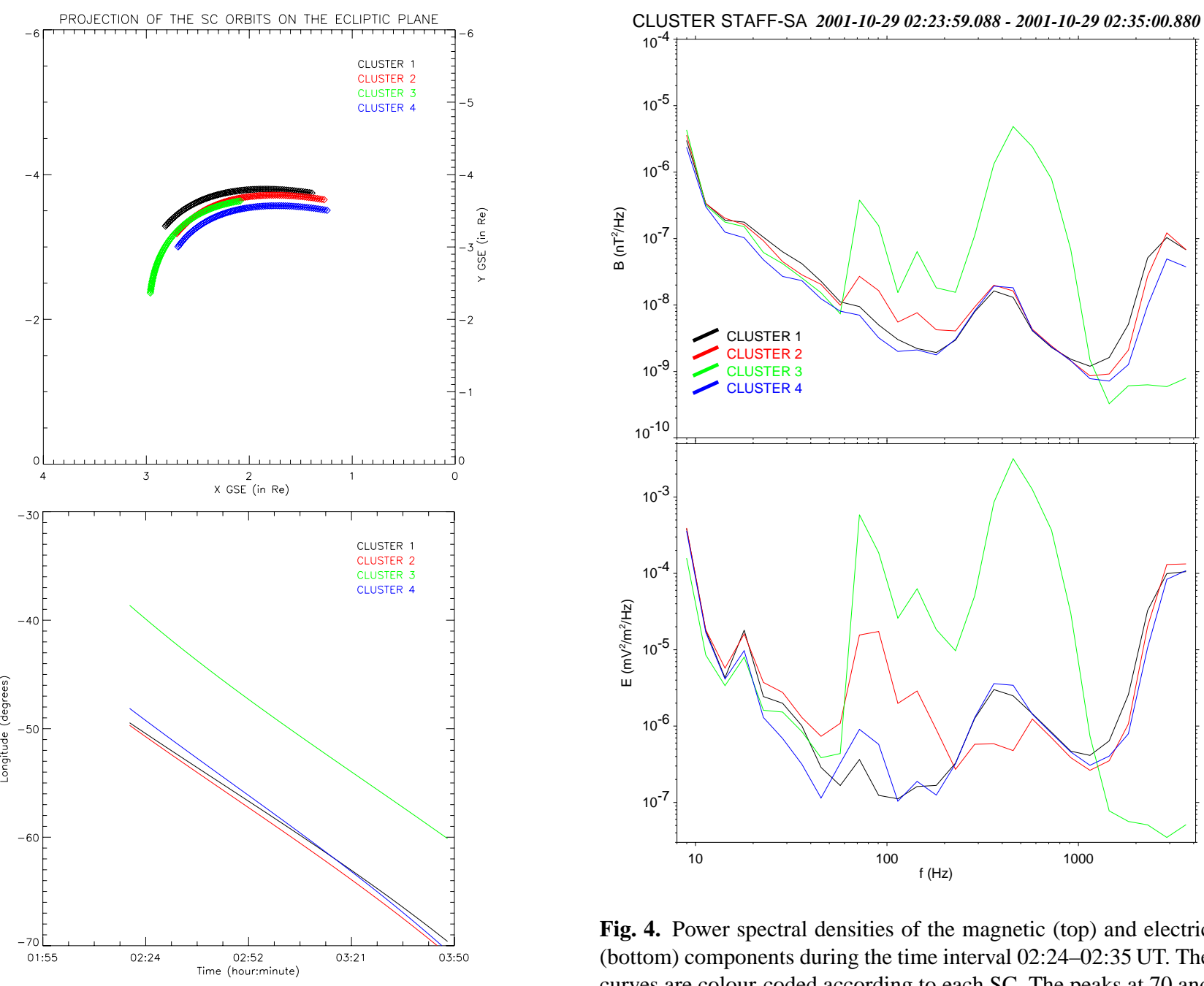

Fig. 4. Power spectral densities of the magnetic (top) and electric (bottom) components during the time interval 02:24-02:35 UT. The curves are colour-coded according to each SC. The peaks at 70 and $140 \mathrm{~Hz}$ are interferences.

Fig. 3. The top panel represents the projection of the SC orbits in the ecliptic plane, whereas the bottom panel shows the corresponding azimuthal angle (longitude) as a function of time in the interval 02:19:30-03:48:30 UT. The curves are colour-coded according to each SC.

The four last panels present propagation characteristics of these emissions in frequency-time plots similar to spectrograms with intensities colour-coded according to the scale on the right. These panels give an estimation of the sign of the parallel component of the Poynting vector relative to the Earth's magnetic field B ${ }_{0}$ (Santolík et al., 2001; Parrot et al., 2003). Geophysical parameters at the bottom of Fig. 1 are related to SC1. In the Poynting vector panels, a blue colour indicates that the wave vector is in the opposite direction of the Earth's magnetic field $\mathrm{B}_{0}$, i.e. directed towards the south, whereas the red colour is related to wave vectors in the direction of $\mathrm{B}_{0}$, i.e. directed towards the north. This assumption is valid for observations at low- and mid-latitudes, which is the case in this event where the magnetic latitude is always less than $43^{\circ}$. It is observed on all SC for the main intense chorus emissions that the colour changes close to the equator, which indicates that the chorus waves propagate away from

the equator. In these four last panels, it is also observed at the same time below the main emissions for each satellite, emissions at lower frequencies, which for most of the time propagate in an opposite direction. These emissions are directed toward the equator, and it is postulated that they are related to the main intense one. This will be demonstrated in the next paragraph using ray tracing techniques.

Figure 2 represents a zoom of Fig. 1 from 02:20 until 03:50 UT, a time interval that will be studied in more details in what follows. The last group of four panels shows again the direction of the Poynting vector for the 4 SC. The first and second group of four panels represent the polar and the azimuthal angles of the wave normal direction relative to the Earth's magnetic field $\mathrm{B}_{0}$ (given by the FGM PP), respectively. These angles are obtained from the magnetic spectral matrix with the method of Means (1972). This method can be used for these emissions because their degree of polarisation (not shown here) obtained from the eigenvalues of the spectral matrices is high enough $(\sim 0.8)$, which is an indication of plane waves (Lefeuvre and Parrot, 1979). Geophysical parameters at the bottom of Fig. 2 are related to SC3. 
Four time intervals have been chosen for the inverse ray tracing study using as inputs the polar and azimuthal angles of the wave normal direction, the local electron density given by the WHISPER experiment, and the relative satellite positions which are given in Fig. 3. Figure 3a represents the projection of the SC orbits in the ecliptic $(X-Y)$ GSE (Geocentric Solar Ecliptic) plane. The curves are colour-coded according to each SC and the time (02:20-03:50 UT) is going from the left to the right. Figure $3 b$ is derived from $3 a$; it gives the azimuthal angle (related to the longitude) in the ecliptic plane as a function of time. At a given time, similar angles for the $\mathrm{SC}$ indicate that these $\mathrm{SC}$ are on the same meridian line.

The first time interval is from 02:24 until 02:35 UT, and Fig. 4 represents the power spectral densities of the 3 magnetic and the 2 electric components in the top and bottom panels, respectively. The plots which represent average values over the 11-min interval, are done as a function of the frequency for each SC, and SC3 shows a peak centred around $456 \mathrm{~Hz}$ according to the intensities observed in Fig. 1 (the peaks around $70 \mathrm{~Hz}$ and $140 \mathrm{~Hz}$ are due to internal interferences of the Spectrum Analyser). At the same time as the peak on SC3, power spectral density increases with much lower intensities are also observed on SC1, SC2, and SC4. Four frequencies of STAFF-SA have been selected for the ray tracing: $456,574,724$, and $912 \mathrm{~Hz}$. Those frequencies are central frequencies of the different equivalent filters. It is seen on the last four panels of Fig. 2 that these frequencies correspond to $\mathrm{SC} 3$ waves escaping from the equator (blue) and to waves going to the equator for $\mathrm{SC} 1, \mathrm{SC} 2$, and $\mathrm{SC} 4$ (red). But it is also observed that the frequency bandwidth of the peaks observed in Fig. 4 does not fit exactly with the frequency bandwidth of the red zone in Fig. 2, and this point will be discussed later. The peak at $2896 \mathrm{~Hz}$ in Fig. 4 for $\mathrm{SC} 1, \mathrm{SC} 2$, and SC4 corresponds to the intense waves coming directly from the equator, which are not studied here.

The results of the multiple ray tracing for each satellite and for each polar and azimuthal determinations of the wave normal direction between 02:24 and 02:35 UT are given in Fig. 5. Figure 5a represents the 3-D plots of the rays in GSM coordinates for $574 \mathrm{~Hz}$. The ray tracing software was stopped when the rays encountered the equator. Rays corresponding to one SC are colour-coded according to the dedicated SC colours seen on the right upper corner. Each line corresponds to a ray at a different time, then trajectories of all SC between 02:24 and 02:35 UT can also be seen on this 3-D plot. They are plotted by the points of departure of each ray when the time is going on. In order to have a better view of the ray trajectories, this 3-D plot has been projected on the 3 planes $X Y, X Z$, and $Y Z$ in Fig. 5b. It is clearly shown on this plot that the waves observed, on one hand, by $\mathrm{SC} 3$, and, on the other hand, by $\mathrm{SC} 1, \mathrm{SC} 2$, and $\mathrm{SC} 4$, span the same region in the equatorial plane (the source region of chorus). Waves that originate from the equator are first seen by SC3. After that, they are going to higher latitudes where they suffer a LHR (Lower Hybrid Resonance) reflection at low altitudes. The reflection occurs when the frequency of the wave approaches the local LHR frequency (Kimura, 1966). At the end, they
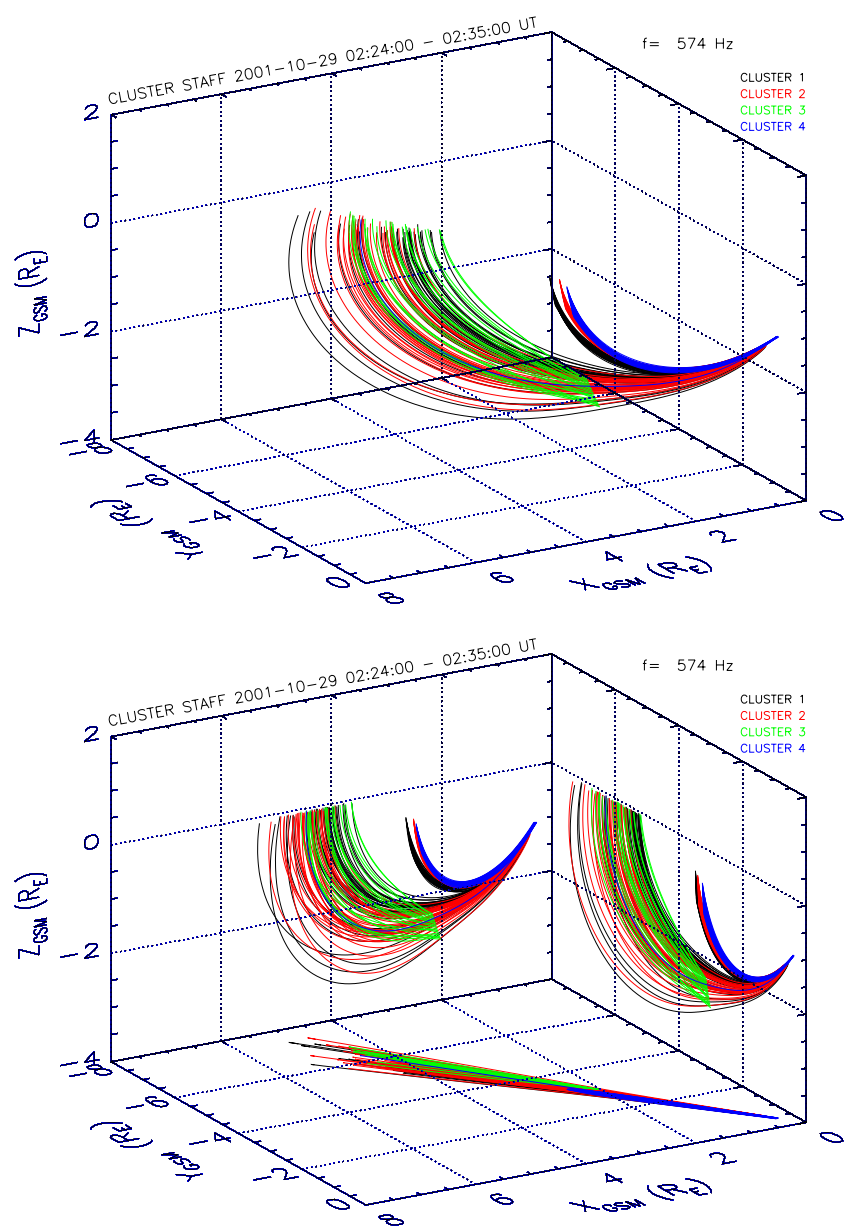

Fig. 5. The top panel represents the ray traces in 3-D for a frequency equal to $574 \mathrm{~Hz}$ during the same time interval as Fig. 4. The bottom panel represents the same data but now only the projections of the ray traces on the three planes are plotted. The curves are colourcoded according to each SC.

return to the equator at a different $\mathrm{L}$ value. On their way back, they are observed by $\mathrm{SC} 1, \mathrm{SC} 2$, and to a lesser extent by $\mathrm{SC} 4$, because a very small number of rays is obtained for this SC. Plots for other frequencies are shown in Fig. 6. They are similar to Fig. 5b and represent ray tracing for 456 and $724 \mathrm{~Hz}$ in Figs. 6a and 6b, respectively. At $456 \mathrm{~Hz}$, it is noted that the waves observed on SC4 map in the equatorial plane to a region that is different from the other SC. This will be discussed in the next section. At $724 \mathrm{~Hz}$, the results are similar to Fig. 5b, however, the extension of the source at the equator diminishes. At $912 \mathrm{~Hz}$, it still decreases (not shown).

The second time interval is from 02:56 until 03:05 UT and the third one is from 03:10 until 03:16UT. SC1, SC2, and SC4 are now in the Northern Hemisphere whereas SC3 is still in the Southern Hemisphere. In the second time interval (02:56-03:05 UT), it is seen from Fig. 2 that SC1, SC2, and SC4 observe waves at frequencies between 724 and $1149 \mathrm{~Hz}$ going toward the equator, whereas SC3 observes the main intense emission at the same frequencies coming from the equator. In the third time interval (03:10-03:16 UT), Fig. 2 

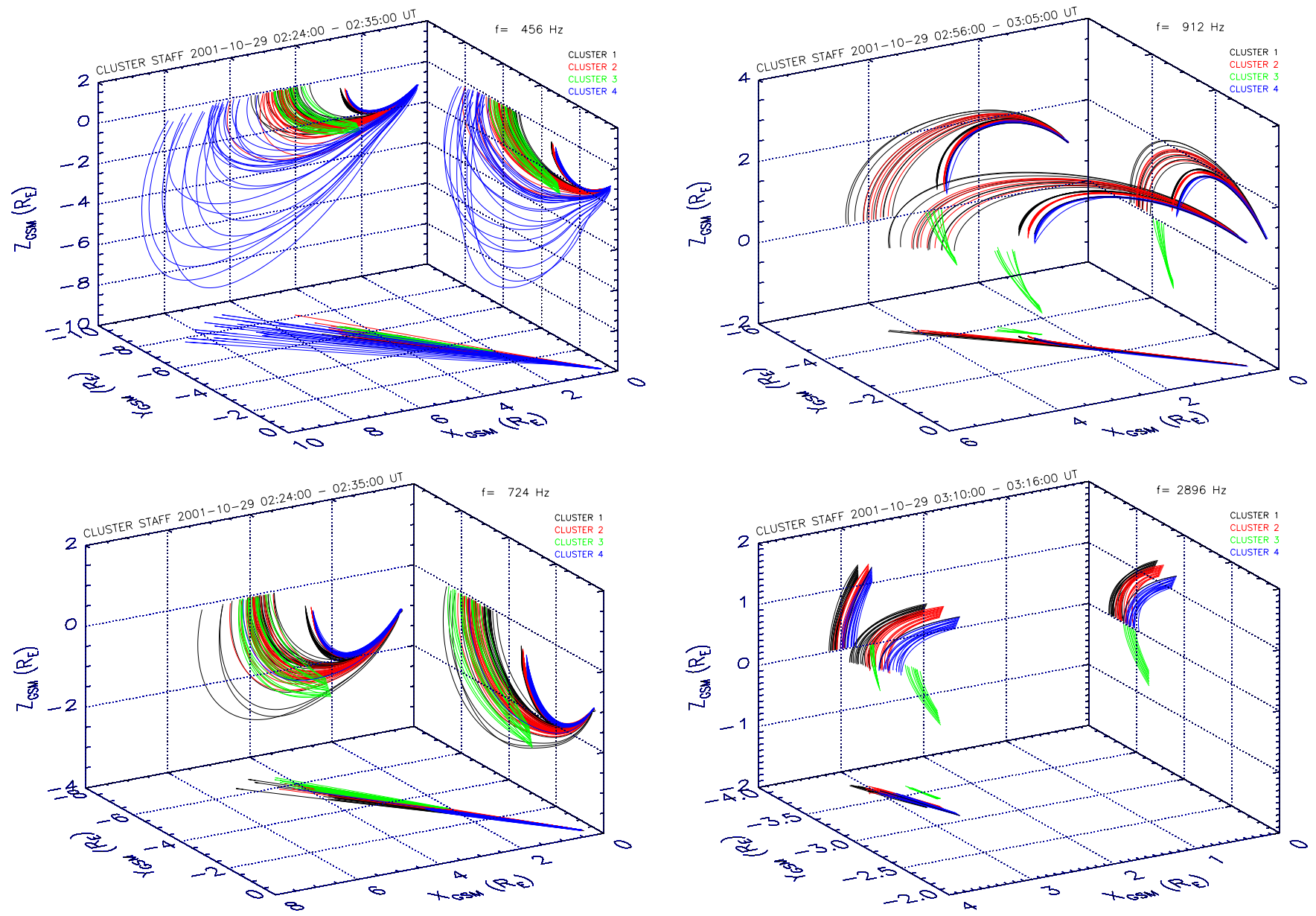

Fig. 6. Same as the bottom panel of Fig. 5, but the top and the bottom panels correspond to 456 and $724 \mathrm{~Hz}$, respectively.

indicates that $\mathrm{SC} 1, \mathrm{SC} 2, \mathrm{SC} 3$ and $\mathrm{SC} 4$ ( $\mathrm{SC} 3$ is still in the Southern Hemisphere) observe their main emissions coming from the equator at frequencies larger than $1824 \mathrm{~Hz}$. Ray plots corresponding to these second and third time intervals are shown in Figs. 7a and b, respectively. They are represented on the plot in 3D together with their projections. Figure 7a shows that $\mathrm{SC} 1, \mathrm{SC} 2$, and $\mathrm{SC} 4$ observe waves coming from the equator after a LHR reflection whereas SC3 observe waves coming directly from the equator. The mapping in the equatorial source region indicates that there is no relation between these waves because the origin of the waves observed by $\mathrm{SC} 3$ does not fit with the origin of the waves observed by $\mathrm{SC} 1, \mathrm{SC} 2$, and $\mathrm{SC} 4$. However $\mathrm{SC} 1, \mathrm{SC} 2$, and $\mathrm{SC} 4$ observe emissions coming from the same region. Figure $7 \mathrm{~b}$ (time interval 03:10-03:16 UT) indicates that all SC observe their more intense emission coming directly from the equator. Waves observed on the one hand by $\mathrm{SC} 1, \mathrm{SC} 2$, and $\mathrm{SC} 4$ and on the other hand by $\mathrm{SC} 3$ are again not coming from the same region because there is no overlap of the source regions in the $X$-direction. At lower frequencies, the SC3 panel of Fig. 2 ( $z$-component of the Poynting vector) also indicates that different waves return to the equator during this

Fig. 7. The ray plots are represented (together with their projections in the three planes) in the top and bottom panels for the time intervals 02:56-03:05 UT and 03:10-03:16 UT, respectively. The top and the bottom panels correspond to $912 \mathrm{~Hz}$ and $2896 \mathrm{~Hz}$, respectively.

third time interval (red area between 700 and $1000 \mathrm{~Hz}$ ). It is an observation similar to the first case but the direct wave is no longer seen on $\mathrm{SC} 1, \mathrm{SC} 2$, and $\mathrm{SC} 4$ at this time.

A fourth time interval has been selected between 03:32 and $03: 42$ UT to study the frequency range $400-1000 \mathrm{~Hz}$. All SC are now in the Northern Hemisphere and it is a counterpart to the first case where all SC were in the Southern Hemisphere. Figure 8, which is similar to Fig. 4, represents the power spectral densities of the 3 magnetic and the 2 electric components in the top and bottom panels, respectively. The plots which represent average values over the $10 \mathrm{~min}$ interval, are done as a function of the frequency for each SC, and $\mathrm{SC} 1, \mathrm{SC} 2$, and $\mathrm{SC} 4$ show a peak centred around $500 \mathrm{~Hz}$ according to the intensities observed in Fig. 1. At the same time as the peak on these three SC, power spectral density increases with much lower intensities are also observed on SC3. Four frequencies of STAFF-SA have been selected for the ray tracing: $456,574,724$, and $912 \mathrm{~Hz}$ (not all shown). It is seen on the last four panels of Fig. 2 that these frequencies correspond to $\mathrm{SC} 1, \mathrm{SC} 2$, and $\mathrm{SC} 4$ waves escaping from 


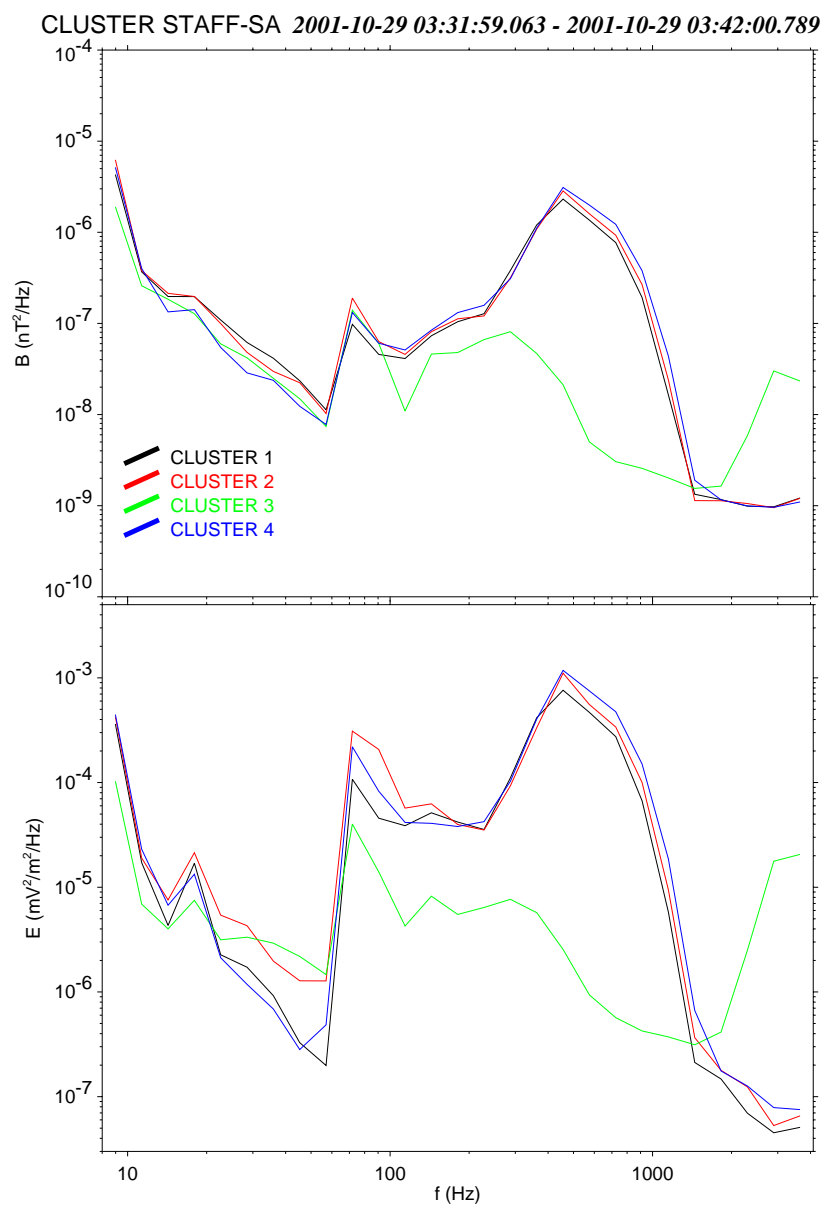

Fig. 8. Same as Fig. 4 for the time interval 03:32-03:42 UT.

the equator (red), whereas they correspond to waves going to the equator for SC3 (blue). It is also observed that the frequency bandwidth of these waves going to the equator for SC3 does not cover exactly the corresponding frequency bandwidth of the peaks observed in Fig. 8, as it did in the first case. The results of the multiple ray tracing for each polar and azimuthal determinations between 03:32 and 03:42 UT are given in Fig. 9 for a frequency equal to $724 \mathrm{~Hz}$. Figure 9 represents the 3-D plots of the rays in GSM coordinates and their corresponding projections on the three planes. The number of SC3 rays is not so important but, as in the first time interval, it is observed that all SC record waves spanning the same equatorial region. SC3 observes these waves after a LHR reflection.

\section{Discussions and conclusion}

The ray tracing shows that, at the same time, direct waves are emitted from the equator (the chorus source region) and reflected waves come through the same equatorial region. The origin of these reflected waves must be the same as the main chorus emission (a hiss emission going through the equator would not have enough intensity to be observed after a re-

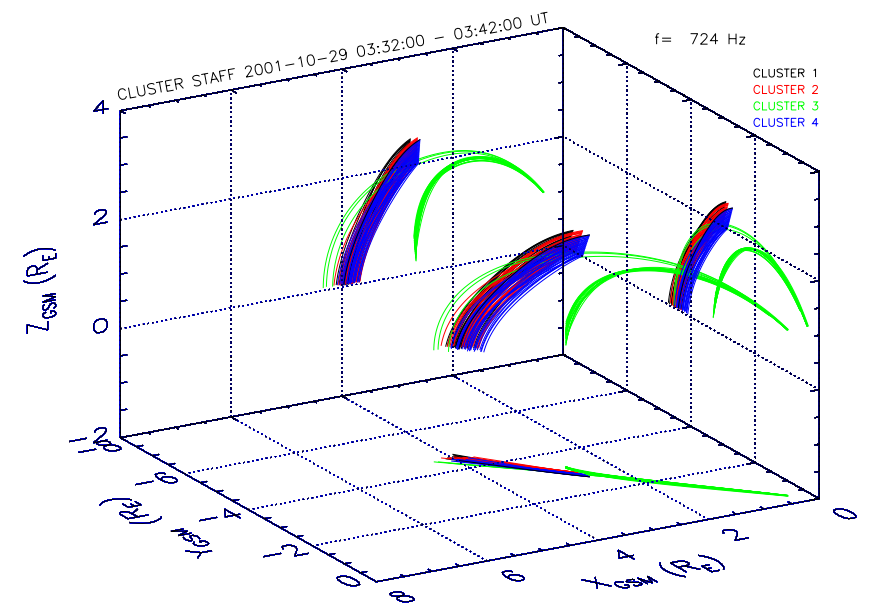

Fig. 9. Plots of the ray traces in 3-D and their corresponding projections on the three planes for the time interval 03:32-03:42 UT and for a frequency equal to $724 \mathrm{~Hz}$.

flection). Then, for the first time it was possible to simultaneously observe waves directly emitted from the source region and the same waves returning to the equator after a LHR reflection. At least two satellites are necessary to find (using the ray tracing) that, for example, in case 1 of Fig. 2, the two emissions seen at the same time, at the same frequency, but not at the same location on SC1 (SC2, or SC4) and SC3 (one being directed toward the equator and the other away from the equator), have the same origin, and then to demonstrate that we have observed a reflected wave. This opportunity is due to the simultaneous data recording on the four CLUSTER SC and also to the particular configuration of their orbits during this day. SC3 was nearly on the same orbit as the others but with a delay of $30 \mathrm{~min}$. Correspondences between the meridian lines of, on the one hand, $\mathrm{SC} 3$ and, on the other, hand $\mathrm{SC} 1, \mathrm{SC} 2$, and $\mathrm{SC} 4$, can also be seen on the $X Y$ GSE planes of Figs. 5b (first time interval) and 9 (fourth time interval) where their traces point in the same direction. It is not the case in Figs. 7a (second time interval) and 7b (third time interval) where SC3 and the other three SCs observe waves coming from different source regions.

The discrepancy that is observed in the first interval between the peak in Fig. 4 located around $456 \mathrm{~Hz}$ and the frequency bandwidth of waves returning to the equator (red area in Fig. 2) can be explained by the experimental configuration of the STAFF-SA experiment (the situation is similar for the fourth interval). This frequency bandwidth in Fig. 2 is comprised between 600 and $1000 \mathrm{~Hz}$, which corresponds to the frequency sub-band of analysis $C$ (see Sect. 2). It means that analysis in this frequency sub-band gives a better spectral matrix estimation due to a larger number of averaged spectra relative to the frequency sub-bands B and A. Due to this fact, the estimation of the wave propagation parameters that are derived from the spectral matrix is also much better in the sub-band $\mathrm{C}$. In this event where the wave maximum occurs in the sub-band $\mathrm{B}$, the reliability of the analysis result 
for the sign of the $z$-component of the Poynting vector is not excellent (mixture of red and yellow values below $512 \mathrm{~Hz}$ in Fig. 2). This difference between the sub-bands $\mathrm{B}$ and $\mathrm{C}$ is clearly seen in the last four panels of Fig. 2.

The fact that in Fig. 6a, SC4 does not map correctly the source at a frequency equal to $456 \mathrm{~Hz}$ can be due to its location relative to $\mathrm{SC} 1$ and $\mathrm{SC} 2$. It is seen in Fig. $3 \mathrm{~b}$ that, at the beginning and especially during the first time interval, SC4 is not exactly on the same meridian line. It could explain that SC4 receives waves coming from a different region.

Cornilleau-Wehrlin et al. (1985) have studied the interaction between energetic electrons and whistler mode ELF/VLF magnetic waves measured by GEOS. They determined the spatial growth rate of these waves and, therefore, they deduced that the corresponding reflection coefficient at the ionosphere for the wave power intensity must be of the order of 0.05 . In the present study, it is possible to experimentally measure this coefficient which is directly given by plots in Figs. 4 and 8. In the first case (Fig. 4), the ratio for the magnetic and electric power spectral densities at $400 \mathrm{~Hz}$ between SC3 and the three others is 0.005 and 0.001 , respectively. The ratio for the fourth case (Fig. 8) is 0.01 and 0.003 , respectively. This of course depends on the plasma parameters along the reflected wave path during this particular day, but it is observed that the experimental magnetic ratio obtained in the two cases fits the theoretical one obtained by Cornilleau-Wehrlin et al. (1985).

Goldstein and Tsurutani (1984) and Hayakawa et al. (1984) have shown that the wave normal of chorus elements at frequencies lower than one-half the electron gyrofrequency is close to the Earth's magnetic field at the equator, which seems to indicate that the generation mechanism could be due to electron cyclotron instability. It is interesting to check the values of the polar angle $\theta$ of waves returning to the equator during this event. Looking at the four bottom panels of Fig. 2, these waves approach the equator in three cases (around $1 \mathrm{kHz}$, blue colour): on $\mathrm{SC} 1$ and $\mathrm{SC} 2$ at $\sim 02: 48$ UT, and on SC3 at 03:28 UT. All cases indicate a similar feature, as it can be seen on the top panels of Fig. 2. When the satellites get closer to the equator the polar angle $\theta$ moves to values less than $20^{\circ}$. Goldstein and Tsurutani (1984) concluded that the wave growth is maximum for waves propagating parallel to the Earth's magnetic field at the magnetic equator where this magnetic field is minimum. Therefore, it can be expected that the waves returning to the equator will endure a further amplification. A more detailed study of the wave normal angle of chorus emissions recorded by CLUSTER will be published elsewhere.

With this ray tracing study it is also possible to evaluate the minimum size of the chorus source. In this event which is at around 08:40 MLT (Magnetic Local Time), the $X$-extension of the source is between 3.5 and $4.5 R_{E}$, and the $Y$-extension is between 4.5 and $6.5 R_{E}$ at a frequency equal to $724 \mathrm{~Hz}$. In a further work, dimensions of the chorus sources at the equator will be extensively studied for different magnetic local times and different magnetic activities.
Acknowledgements. Thanks are due to the Pis of the FGM (A. Balogh), WHISPER (P. Décreau) and EFW (M. André) experiments for the use of their data, and to the Hungarian Cluster Data Center, which provides auxiliary data. ESA and CNES are thanked for their support for the STAFF experiment.

Topical Editor T. Pulkkinen thanks J. Pickett and another referee for their help in evaluating this paper.

\section{References}

Balogh, A., Dunlop, M. W., Cowley, S. W. H., Southwood, D. J., Thomlinson, J. G., Glassmeier, K. H., Musmann, G., Lühr, H., Buchert, S., Acuña, M. H., Fairfield, D. H., Slavin, J. A., Riedler, W., Schwingenschuh, K., Kivelson, M. G., and the Cluster magnetometer team: The Cluster magnetic field investigation, Space Science Rev., 79, 65-91, 1997.

Burtis, W. J. and Helliwell, R. A.: Banded chorus: A new type of VLF radiation observed in the magnetosphere by OGO-1 and OGO-3, J. Geophys. Res., 74, 3002-3010, 1969.

Burton, R. K. and Holzer, R. E.: The origin and propagation of chorus in the outer magnetosphere, J. Geophys. Res., 79, 10141023, 1974.

Cairo, L. and Lefeuvre, F.: Localization of sources of ELF/VLF hiss observed in the magnetosphere: Three-dimensional ray tracing, J. Geophys. Res., 91, 4352-4364, 1986.

Cerisier, J. C.: Propagation perpendiculaire au voisinage de la fréquence de la résonance hybride basse, in Plasma Waves in Space and in the Laboratory, 2, pp. 487-521, Edinburgh University Press, Edinburgh, 1970.

Cornilleau-Wehrlin, N., Solomon, J., Korth, A., and Kremser, G.: Experimental study of the relationship between energetic electrons and ELF waves observed on GEOS: A support to quasilinear theory, J. Geophys. Res., 90 (A5), 4141-4154, 1985.

Cornilleau-Wehrlin, N., Chauveau, P., Louis, S., Meyer, A., Nappa, J. M., Perraut, S., Rezeau, L., Robert, P., Roux, A., de Villedary, C., de Conchy, Y., Friel, L., Harvey, C. C., Hubert, D., Lacombe, C., Manning, R., Wouters, F., Lefeuvre, F., Parrot, M., Pinçon, J. L., Poirier, B., Kofman, W., Louarn, P., and the STAFF investigator team: The CLUSTER STAFF experiment (Spatio Temporal Analysis of Field Fluctuations Experiment), Space Science Rev., 79, 107-136, 1997.

Cornilleau-Wehrlin, N., Chanteur, G., Rezeau, L., Parrot, M., Pinçon, J. L., and STAFF team: The CLUSTER STAFF experiment specific multi-point analysis, Proc. CLUSTER-II Workshop on Multiscale / Multipoint Plasma Measurements, London, 22-24 September 1999, ESA SP-449, February 2000.

Cornilleau-Wehrlin, N., Chanteur, G., Perraut, S., Rezeau, L., Robert, P., Roux, A., de Villedary, C., Canu, P., Maksimovic, M., de Conchy, Y., Hubert, D., Lacombe, C., Lefeuvre, F., Parrot, M., Pinçon, J. L., Décréau, P. M. E., Harvey, C. C., Louarn, Ph., Kofman, W., Santolík, O., Alleyne, H. St. C., Roth, M., and STAFF team: First results obtained by the Cluster STAFF experiment, Ann. Geophysicae, 21, 437-456, 2003.

Daly, P. W.: Users guide to the CLUSTER Science Data System, DS-MPA-TN-0015, 19 April 2002, ftp://ftp.estec.esa.nl/ pub/csds/task_for/users_guide/csds_guide.html, 2002.

Décréau, P. M. E., Fergeau, P., Krasnosels'kikh, V., Lévêque, M., Martin, Ph., Randriamboarison, O., Sené, F. X., Trotignon, J. G., Canu, P., Mögensen, P. B., and the Whisper experimenters: Whisper, a resonance sounder and wave analyser: performances 
and perspectives for the Cluster mission, Space Sci. Rev., 79, 157-193, 1997.

Décreau, P. M. E., Fergeau, P., Krasnoselskikh, V., Le Guirriec, E., Lévêque, M., Martin, Ph., Randriamboarison, O., Rauch, J. L., Sené, F. X., Séran, H. C., Trotignon, J. G., Canu, P., Cornilleau, N., de Féraudy, H., Alleyne, H., Yearby, K., Mögensen, P. B., Gustafsson, G., André, M., Gurnett, D. C., Darrouzet, F., Lemaire, J., Harvey, C. C., Travnicek, P., and Whisper experimenters: Early results from the Whisper instrument on Cluster: an overview, Ann. Geophysicae, 19, 1241-1258, 2001.

Dunkel, N. and Helliwell, R.: Whistler mode emissions in the OGO 1 satellite, J. Geophys. Res., 74, 6371-6385, 1969.

Goldstein, B. E. and Tsurutani, B. T.: Wave normal directions of chorus near the equatorial source region, J. Geophys. Res., 89, 2789-2810, 1984.

Gustafsson, G., André, M., Carozzi, T., Eriksson, A. I., Fälthammar, C.-G., Grard, R., Holmgren, G., Holtet, J. A., Ivchenko, N., Karlsson, T., Khotyaintsev, Y., Klimov, S., Laakso, H., Lindqvist, P.-A., Lybekk, B., Marklund, G., Mozer, F., Mursula, K., Pedersen, A., Popielawska, B., Savin, S., Stasiewicz, K., Tanskanen, P., Vaivads, A., and Wahlund, J.-E.: First results of electric field and density observations by Cluster EFW based on initial months of operation, Ann. Geophysicae, 19, 1219-1240, 2001.

Hayakawa, M., Yamanaka, Y., Parrot, M., and Lefeuvre, F.: The wave normals of magnetospheric chorus emissions observed on board GEOS 2, J. Geophys. Res., 89, 2811-2821, 1984.

Kennel, C. F. and Petscheck, H. E.: Limit on stably trapped particle fluxes, J. Geophys. Res., 71, 1-28, 1966.

Kimura, I.: Effects of ions on whistler-mode ray tracing, Radio Science, 1(3), 269-283, 1966.

LeDocq, M. J., Gurnett, D. A., and Hospodarsky, G. B.: Chorus source locations from VLF Poynting flux measurements with the Polar spacecraft, Geophys. Res. Lett., 25 (21), 4063-4066, 1998.

Lefeuvre F. and Parrot, M.: The use of the coherence function for the automatic recognition of chorus and hiss observed by GEOS1, J. Atmos. Terr. Phys., 41, 143-152, 1979.

Means, J. D.: Use of the three-dimensional covariance matrix in analyzing the polarisation properties of plane waves, J. Geophys. Res., 77, 5551-5559, 1972.

Meredith, N. P., Horne, R. B., and Anderson, R. R.: Substorm dependence of chorus amplitudes: Implications for the acceleration of electrons to relativistic energies, J. Geophys. Res., 106,
13 165-13 178, 2001.

Muto, H. and Hayakawa, M.: Ray-tracing study of the propagation in the magnetosphere of whistler-mode VLF emissions with frequency above one half the gyrofrequency, Planet. Space Sci., 35, 1397-1404, 1987.

Nagano, I., Yagitani, S., Kojima, H., and Matsumoto, H.: Analysis of wave normal and Poynting vectors of the chorus emissions observed by Geotail, J. Geomagn. Geoelectr., 48, 299-307, 1996.

Parrot, M., Santolík, O., Cornilleau-Wehrlin, N., Maksimovic, M., and Harvey, C. C.: Source location of chorus emissions observed by CLUSTER, Ann. Geophysicae, 21, 473-480, 2003.

Pedersen, A., Cornilleau-Wehrlin, N., de la Porte, B., Roux, A., Bouabdellah, A., Décréau, P. M. E., Lefeuvre, F., Sené, F. X., Gurnett, D., Huff, R., Gustafsson, G., Holmgren, G., Woolliscroft, L. J. C., Thompson, J. A., and Davies, P. H. N.: The Wave Experiment Consortium (WEC), Space Sci. Rev., 79, 93-106, 1997.

Santolík, O.: Propagation Analysis of Staff-SA Data with Coherency Tests, LPCE/NTS/073.C, Lab. Phys. Chimie Environ./CNRS, Orléans, France, 2001.

Santolík, O. and Parrot, M.: Propagation analysis of electromagnetic waves between the helium and proton gyro-frequencies in the low-altitude auroral zone, J. Geophys. Res., 103, 20469 $20480,1998$.

Santolík, O. and Parrot, M.: Case studies on wave propagation and polarisation of ELF emissions observed by Freja around the local proton gyro-frequency, J. Geophys. Res., 104, 2459-2476, 1999.

Santolík, O., Lefeuvre, F., Parrot, M., and Rauch, J. L.: Complete wave-vector directions of electromagnetic emissions: Applications to INTERBALL-2 measurements in the night-side auroral zone, J. Geophys. Res., 106, 13 191-13 201, 2001.

Sazhin, S. S. and Hayakawa, M.: Magnetospheric chorus emissions: A review, Planet. Space Sci., 40, 681-697, 1992.

Tsurutani, B. T. and Smith, E. J.: Postmidnight chorus: a substorm phenomenon, J. Geophys. Res., 79, 118-127, 1974.

Tsurutani, B. T. and Smith, E. J.: Two types of magnetospheric ELF chorus and their substorm dependences, J. Geophys. Res., 82, 5112-5128, 1977.

Tsurutani, B. T., Smith, E. J., West, Jr., H. I., and Buck, R. M.: Chorus, Energetic electrons and magnetospheric substorms, in: Wave Instabilities in Space Plasmas, (Eds) Palmadesso, P. J. and Papadopoulos, K., D. Reidel Publishing Company, 55-62, 1979. 